\title{
DIABETES IN PALLIATIVE CARE
}

Fawad Ahmad (Staff Grade Doctor) and Carey Mills (Blue Team Leader) Supervised by: Dr Simone Ali (Consultant Palliative Medicine)

\section{Introduction}

Diabetes affects about 171 million people worldwide. Current UK prevalence of diabetes in people over 65 is 10\% .In 2005 about 10 million people were diagnosed with cancer. The incidence of diabetes in patients with cancer is postulated to be higher when compared to the general population.

Causes of diabetes in patients with advanced disease are multifactorial and include increased incidence, advanced cancer (i.e. pancreatic), obesity, metabolic changes due to cancer and use of diabetogenic drugs such as corticosteroids, octreotide and diuretics.

Careful monitoring and control of blood glucose levels via diet and glucose lowering medicines are essential components of glycemic control. Evidence based guidelines exist for management of patients with diabetes but glycemic management in context of advanced cancer varies and lack a suitable evidence base.

\section{Aetiology and Presentation}

Diabetes can be primary or secondary. Primary diabetes is where no underlying cause can be identified. Secondary diabetes occurs when there is underlying pancreatic disease (pancreatectomy, malignancy and pancreatitis), endocrine disorder (Cushing's, acromegaly) or is drug induced.

The diagnosis of diabetes has already been made in the majority of patients who are referred to palliative care services. However a number of patients will either be diagnosed or develop diabetes as a result of the treatment.

Commonly presenting symptoms are polyuria, polydipsia, fatigue and weight loss. A small number of patients present acutely with hyperglycaemic emergencies.

\section{Diagnosis and Monitoring}

WHO criteria for diagnosing diabetes are as follows:

1) In asymptomatic patients, fasting blood sugar measurement of $7 \mathrm{mmol} / \mathrm{L}$ and more or random blood sugar measurement of $11 \mathrm{mmol} / \mathrm{L}$ and more on two separate occasions.

2) In symptomatic patients, random blood sugar measurement of more than 11 $\mathrm{mmol} / \mathrm{L}$.

3) For borderline patients, definitive diagnosis can be made using a glucose tolerance test.

Further monitoring in palliative care patients depends upon the stage of underlying disease. In early stages, diabetic patients should be monitored normally. Those with advanced disease should be monitored in context of symptom control.

Measurement of capillary blood glucose is most convenient and appropriate test for continuous monitoring. Glycated haemoglobin indicated control over 2-3 month period. Urinalysis has limited sensitivity, specificity and cannot detect hypoglycaemia. 


\section{Management Issues}

Management of diabetes in patients with advanced cancer is complicated by a number of factors. Blood sugar control depends upon a balance between hypoglycaemic medication and food intake. Therefore anorexia associated with malignancy affects glycemic control. Other factors include nausea and vomiting associated with malignancy or Opioid analgesics, deranged GI motility and obstruction resulting in poor absorption and medication such as diuretics and steroids used for symptom control.

Management of diabetes may be further complicated because glucose intolerance is one of the first metabolic consequences of cancer. There is insulin resistance with increased hepatic glucose production, reduced glucose utilization by skeletal muscle and reduced skeletal muscle glycogen synthesis.

\section{General Principles of Management}

- During the early stage of palliative care where the person may still be active and have possibly many years left to live, there is no reason why diabetes should not be managed conventionally.

- As disease progresses and prognosis becomes short term, the importance of preventing the long-term complications of diabetes become less significant. Both hyperglycaemia (ketoacidosis, non-osmolar coma) and hypoglycaemia are uncomfortable, unpleasant and should be avoided. Maintaining a blood glucose level between 8-15 mmol/L rather than absolute values is important.

- It is important to explain patients and families why a more relaxed approach is being taken as they may perceive this as giving up on the patient.

- Intensity of monitoring depends upon the expected prognosis.

- Periodic measurement of blood glucose should be done on all patients started on steroids particularly if patients develop symptoms suggestive of hyperglycaemia.

\section{Management}

\section{1) Type 1 or Type 2 insulin controlled}

\section{a) Early phase}

\section{Range}

- Acceptable range: 8-15 mmol/L.

- If $\mathrm{BM}>15 \mathrm{mmol} / \mathrm{L}$ only treat if symptomatic. Administer up to 5 units of short acting insulin, e.g. novorapid. Recheck BM after an hour; if BM has remained above $15 \mathrm{mmols} / \mathrm{L}$ only treat if symptomatic.

\section{Monitoring}

- As per usual regime i.e. up to twice a day. Medication

- Administer Insulin as per usual regime. Note: Adjust frequency of monitoring and medication depending on condition e.g. decreased dietary intake, nausea and vomiting.

\section{b) Terminal phase}


Range

- Acceptable range 8-15 mmol/L

- If $\mathrm{BM}>15 \mathrm{mmol} / \mathrm{L}$ only treat if symptomatic, administer up to 5 units of short acting insulin, e.g. Novorapid. Recheck BM after an hour; if BM has remained above $15 \mathrm{mmol} / \mathrm{L}$ only treat if symptomatic.

Monitoring

- Daily pre-insulin BM for 2 days, if asymptomatic and BM> 4 stop all monitoring. Another option is to continue monitoring BM once a day.

\section{Medication}

- Ideally convert to once daily Lantus or twice daily Mixtard. Dose would be up to $1 / 2$ or $2 / 3^{\text {rd }}$ of total insulin dose previously taken. If needed contact Diabetes Specialist Nurses at RSCH for advice.

- Patients who are unconscious or near death, administration of insulin may be unnecessary because they will not experience the symptoms of hyperglycaemia at this stage.

\section{2) Type 2, Tablet or Diet controlled}

\section{a) Early phase}

\section{$\underline{\text { Range }}$}

- Acceptable range 8-15 mmol/L

Monitoring

- Adjust as dictated by condition. Normally glucose levels to be checked up to twice a week if stable levels seen.

\section{Medication}

- Ideally patients should be on short acting sulphonylureas as Gliclazide because it reduces the incidence of adverse effects of other oral agents.

- Normal dose should be continued for as long as possible. However reduced dose may be needed because of decreasing oral intake. Concurrent use of diabetogenic drugs as steroids may require the dose to be increased.

- With deteriorating diabetes control (persistently >15 mmol/L), covert to insulin.

- Adjust as dictated by condition.

\section{b) Terminal phase}

- Stop dietary restrictions, monitoring and medication.

\section{References}

1) British Diabetic Association-- Guidelines of practice for residents with diabetes in care homes. 1999

2) Boyd K. Diabetes Mellitus in Hospice patients: some guidelines. Palliative medicine 1993; 7:163-164 
3) Poulson J. The management of diabetes in patients with advanced cancer. J pain symptom management 1997; 13(6):339-346

4) Ford-Dunn S., Quin J. Management of diabetes during the last days of life. Palliative medicine 2006; 20:197-203

5) Usborne C., Wilding J. Treating diabetes mellitus in palliative care patients. European journal of palliative care $2003 ; 10(5): 186-188$

6) McCann M., White C., Watson M. Practical management of Diabetes Mellitus. European journal of palliative care 2006; 13(6):226-229

7) Quinn K., Hudson P. Diabetes Management in patients receiving palliative care. J pain symptom management 2006; 32(3):275-285

8) Smyth T., Smyth D. How to manage diabetes in advanced terminal illness. Nursing times 2005; 101(17):30-32

9) Tookman A., Wilding J. Guidelines for the management of Diabetes in palliative care. Merseyside and Cheshire Palliative care network Audit group Jan 2003. 